\title{
Use of ultrasound in image-guided high-dose-rate brachytherapy: enumerations and arguments
}

\author{
Susovan Banerjee, MD, Tejinder Kataria, MD, DNB, Deepak Gupta, MD, Shikha Goyal, MD, DNB, \\ Shyam Singh Bisht, MD, Trinanjan Basu, MD, Ashu Abhishek, MD \\ Department of Radiation Oncology, Medanta - The Medicity, Gurgaon, Haryana, India
}

\begin{abstract}
Inherently, brachytherapy is the most conformal radiotherapeutic technique. As an aid to brachytherapy, ultrasonography (USG) serves as a portable, inexpensive, and simple to use method allowing for accurate, reproducible, and adaptive treatments. Some newer brachytherapy planning systems have incorporated USG as the sole imaging modality. Ultrasonography has been successfully used to place applicator and dose planning for prostate, cervix, and anal canal cancers. It can guide placement of brachytherapy catheters for all other sites like breast, skin, and head and neck cancers. Traditional USG has a few limitations, but recent advances such as 3-dimensional (3D) USG and contrast USG have enhanced its potential as a dependable guide in high-dose-rate image-guided brachytherapy (HDR-IGBT). The authors in this review have attempted to enumerate various aspects of USG in brachytherapy, highlighting its use across various sites.

Key words: image guided brachytherapy, high-dose-rate, ultrasonography.

\section{Purpose}

Brachytherapy, owing to the close apposition between the target and the radiation source, is undoubtedly the radiotherapeutic technique that offers maximum conformality. Ultrasound (USG) makes use of the different echogenicity between different tissues, and serves as an effective tool for delineating tumors and differentiates them from surrounding normal tissues. Ultrasonography is attractive as an image guidance modality in brachytherapy, since it is portable, inexpensive, and simple to use, and thus allows for accurate, conformal, reproducible, and adaptive treatments. The newer planning systems for brachytherapy utilize USG as the sole modality for imaging. An imaging based brachytherapy procedure can be divided into four different steps: 1) image-assisted provisional treatment planning, 2) image-guided application, 3) image-assisted definitive treatment planning, 4) image-assisted quality control. European Society for Radiotherapy and Oncology (ESTRO) has recognized USG as a valuable tool for the first 3 steps [1]. Considering the widespread use of USG in prostate cancer brachytherapy and its increasing role in gynecological and breast cancer brachytherapy, we decided to explore the current status of its use in brachytherapy in all sites, recent advances, and future possibilities.

\section{Material and methods}

English language literature (till November 2016), illustrating the use of USG for brachytherapy, was queried for the following terms: 'brachytherapy', 'high-dose-rate', 'ultrasound', '3-dimensional ultrasound', 'image guidance', 'planning for individual sites of cancer'. The available evidences were summarized, including indications from more widespread and routine use in clinical practice to less common sites. The focus was not to give a systematic review, but to include studies that help sensitize the reader about varied uses and development of USG for brachytherapy in different sites.

\section{Ultrasonography imaging in prostate brachytherapy}

In the 1980s, the use of transperineal needles and transrectal USG (TRUS) guidance brought attention to brachytherapy for prostate cancer [2]. The use of USG gathered momentum with its widespread use in lowdose-rate (LDR) pre-planning, LDR seed insertion, and high-dose-rate (HDR) needle or catheter insertion. With continual development, USG overtook all imaging modalities through all steps of prostate brachytherapy. After HDR brachytherapy has shown equivalent clinical results to LDR brachytherapy, it has gained ever increas- 
ing popularity with lesser number of high-dose fractions. Concurrently, the need to accurately deliver the planned treatment with minimal catheter displacement has also arisen. Evolution of TRUS and the availability of sophisticated treatment planning computers have evolved into a reliable system of treating prostate cancer patients by brachytherapy, either in form of boost or monotherapy. A technical note by Morton argues in favor of TRUSbased planning with delivery of a single HDR fraction as an effective and well-tolerated tool for local dose escalation for patients with intermediate and high-risk disease [3]. A TRUS-guided HDR prostate procedure is easy to perform and convenient for the patient. The procedure takes about 90 minutes and the patient is treated without any displacement. Identifying the catheters is not difficult, and a number of USG base planning systems are now commercially available. Early results (median follow-up of 10 months) of use of HDR single fraction boost brachytherapy with use of USG only for positioning and planning have been published by Oliver et al., with dose planning objectives reached in $90 \%$ cases, with none reporting more than grade 2 toxicity, early recovery of baseline urinary function, and promising PSA responses [4]. Recent advances in the field of contrast-enhanced USG seems to be promising for intraprostatic lesion localization as a support for brachytherapy treatment planning. Dose coverage in the intraprostatic lesion could be improved without increasing a dose to organs at risk [5].

\section{Ultrasonography imaging in gynecologic brachytherapy}

Use of USG for gynecologic brachytherapy dates back to the 1970s [6]. The use of real time trans-abdominal ultrasound (TAUS) or TRUS, increases the probability and safety of accurate placement of the uterine tandem. Transabdominal ultrasound performed during an insertion of uterine tandem and ovoids, allows accurate 3D measurement of uterine wall thickness at multiple anatomical levels [7]. This information can be used in conjunction with orthogonal radiographs in order to calculate bladder base doses, and allows real-time assessment of surrounding visceral doses. Ultrasonography has been successfully used for planning in the intracavitary applications incorporating all these advantages [8,9].

One of the first reported uses of USG for interstitial gynecologic brachytherapy was by Stock et al. who demonstrated the usefulness of USG to visualize the target as well as normal tissues in 12 patients [10]. Sharma et al. reported on their experience using TRUS to assist with needle placement in 40 procedures in 25 patients, avoiding any bladder or rectal perforations [11]. Other groups have also reported their experience using USG to assist the guidance of brachytherapy catheters with each demonstrating feasibility and safety in a prospective setting. Investigators from Mumbai have shown a reasonable correlation between TAUS and MRI-based (magnetic resonance imaging) planning [12]. The Melbourne group has used USG as the sole modality for positioning and planning in intracavitary treatment of cancer cervix. Encouraging clinical results in a large cohort of cervical cancer patients (292 patients, non-stage IV) have been reported with a median follow-up period of 4.1 years, a five-year local control of $87.5 \%$, and acceptable toxicity profile. The 5-year failure-free survival and overall survival were $66 \%$ and $65 \%$, respectively [ 8$]$.

Mitchell et al. have described USG based gynecologic planning to be a "2.5-dimensional" approach in the sense that the USG measurements are used to inform the planning, however the prescription is not based on a 3-dimensional volume [13]. Currently, USG cannot be used as a planning tool for complicated interstitial brachytherapy. It can be used to place the needles and delineate the Groupe Européen de Curiethérapie European Society for Radiotherapy and Oncology (GEC-ESTRO) defined highrisk clinical target volume (HR-CTV). The Vienna group suggests that delineating of HR-CTV from TRUS may soon be an acceptable modality [14]. A recent proof of concept has been enunciated for use of trans-cervical endosonography (TRACE) in pre-planning, real-time guidance, and treatment planning of cervical cancer image-guided adaptive brachytherapy (IGABT), and can serve as an interesting addition to complement existing imaging technologies [15].

\section{Ultrasonography imaging in breast brachytherapy}

Interstitial brachytherapy-based accelerated partial breast irradiation (APBI) is a relatively recent development in breast radiotherapy that has gained international favor, with recent evidences suggesting that HDR APBI is here to stay [16]. Interstitial brachytherapy has also been used as a boost therapy after whole breast external beam radiotherapy (EBRT). The first use of USG for APBI was described as early as 1997, and it has now become the most ubiquitous modality used for postoperative applicator placement $[17,18]$. When the applicator is placed in the operating room, USG can also confirm applicator positioning and the lack of air within the lumpectomy cavity [19]. Using three-dimensional ultrasound (3D-USG) to image the patient in breast brachytherapy can be effective as a primary treatment planning imaging modality in breast cancer. The images collected with the applicator in situ can provide a means of optimizing the dose delivered, sparing adjacent normal structures along with a fast and efficient way of delivering personalized real-time guidance, and planning for breast HDR brachytherapy treatments $[20,21]$.

\section{Ultrasonography imaging in anal cancer brachytherapy}

3D endoluminal USG has gained popularity in assessment and interventions for luminal structures such as esophagus and rectum/anal canal. A few centers have extended this application for guiding brachytherapy procedures in anal canal, with the advantages of optimizing implant procedure and better information for dose planning, akin to prostate cancer interstitial brachytherapy. By using an anal obturator or dilator, the dose to the healthy side of the anal canal is limited. Under USG control, the needles are implanted directly into the tumor or tumor bed, and real-time treatment planning is possible. 
Imaging allows for control of the dose distribution and ensures that the whole tumor is covered by the reference isodose [22,23]. Kiel et al. have described their long term clinical experience with this technique in 104 patients with a 10-year mean follow-up period, showing a local control of $89 \%$ and overall survival of $93 \%$ [24].

\section{Lung brachytherapy}

Interstitial brachytherapy has shown promising results for inoperable peripheral lung tumors in terms of safety and efficacy in limited reports, and could potentially be an approach for selected curative treatments, as well as an alternative to radiofrequency ablation, sparing major damage to radiosensitive surrounding structures. In a pilot study reported by Harms et al., inoperable peripheral malignant lung lesions were approached by electromagnetic navigation and the position was confirmed by endobronchial USG [25].

\section{Brachytherapy for abdominal wall metastases}

Kishi et al. have reported on the use of interstitial brachytherapy for treatment of subcutaneous metastatic abdominal wall tumors. They developed a technique utilizing USG-guided insertion of applicator needles to avoid the organs at risk such as intestines, and saline injection into the subcutaneous tissue between the tumor and the skin to decrease the skin dose. Single dose of 20 Gy was administered. Long-lasting pain reduction and tumor shrinkage was obtained without significant early or late complications [26].

\section{Extrahepatic bile duct intraluminal brachytherapy}

Intraluminal brachytherapy with a transcatheter Iridium-192 ( ${ }^{192}$ Ir) wire, as a method to deliver a boost to patients with tumors of extrahepatic bile ducts, has been sufficiently discussed in published series. A major limitation of current imaging techniques is the inability to accurately define the proximal, distal, and most importantly, the lateral extent of tumor. Minsky et al. were able to utilize the advantage of small diameters of endoluminal USG probes allowing insertion into a biliary duct drainage tube and used it to successfully determine tumor volume in the bile ducts [27].

\section{Brachytherapy of head and neck tumors}

Literature on use of USG for head and neck brachytherapy is relatively sparse, but it can be a valuable companion in locally-advanced primary cancers, as well as in recurrent head and neck cancers. Needles can be positioned under continuous USG guidance, and adjacent structures (e.g. the carotid artery) are easily localized, thus avoiding violation of large vessels and optimizing exact positioning of needles within the tumor [28].

\section{Discussion}

Most technical arguments against the use of brachytherapy list the possibility of needle displacement from the time of insertion to the time of treatment, difficulty in delineation of tumor due to artefacts from metallic needles in computed tomography (CT)-based planning, and fear of violating major vessels in the vicinity of tumor. Use of CT-compatible needles as well as magnetic resonance imaging (MRI) based planning have been able to avoid the problem of CT artefacts. However, associated increased cost, and limited availability and training requirements are issues that pose hindrances.

In the era of intensity modulated radiotherapy and stereotactic body radiotherapy, brachytherapy needs to prove itself as a worthy competitor or alternative to external beam radiotherapy for delivering high fractional dose for radical or boost treatments. This is achievable when employing modern imaging tools for guidance and navigation for brachytherapy implantation, as well as for treatment planning and delivery in the same position. This allows to deliver a high dose inside the tumor, sparing surrounding critical structures with zero geometric uncertainty. The American Association of Physicists in Medicine Task Group 43 (AAPM TG-43) dose calculation formalism for photon-emitting radionuclide sources is the mainstay of brachytherapy dosimetry in clinical practice. The TG-43 adopted universally, provides a standardized method to brachytherapy dose calculation based on the superposition of single-source dosimetry in water medium and is simple to use.

The establishment of brachytherapy as the treatment of choice for early organ-confined prostate cancer, where USG imaging for pre- and intraoperative planning and needle insertion along with CT based planning, led to developments in the field of imaging-based brachytherapy treatment planning. This has also translated into similar advances for other sites as well. Conventional USG has been widely used in brachytherapy, mainly to guide needle placement and is useful in planning when combined with other modalities. Two-dimensional viewing of 3-dimensional structures, using conventional USG, limits ability to quantify and visualize the anatomy and guide therapy. It needs multiple 2-dimensional images to be integrated mentally, and increased the possibility of inter-observer variability and inaccuracy [29]. The newer developments in USG such as 3D USG, contrast-enhanced USG, and high frequency USG have been introduced with success in diagnostic, interventional, and therapeutic fields. The 3D USG system gives a spatially oriented and standardized view, thereby reducing operator dependence and improving reproducibility. It gives an accurate $3 \mathrm{D}$ anatomical display and is valuable in carrying interventional procedures by using multiplanar views [29]. In 3D USG imaging, the inter-plane distance can be as low as $1.0 \mathrm{~mm}$, resulting in accuracy better than $1.0 \mathrm{~mm}(\sim 0.5 \mathrm{~mm})$ for catheter reconstruction. Other benefit of USG imaging for the reconstruction of catheters is the possibility to combine $3 \mathrm{D}$ volume reconstruction with live 2D imaging [30]. The United States Food and Drug Administration (US FDA) has not approved a microbubble agent for radiology imaging, but ultrasound contrast agents (UCAs) are popular and widely used in Europe and Asia [31]. Use of contrast-enhanced ultrasound is cost-effective, can be performed at bedside, uses no ion- 
Table 1. Computed tomography, magnetic resonance imaging and ultrasonography in image-guided brachytherapy (authors' recommendations)

\begin{tabular}{lcccccc}
$\begin{array}{l}\text { Imaging } \\
\text { modality }\end{array}$ & $\begin{array}{c}\text { Soft tissue } \\
\text { imaging }\end{array}$ & $\begin{array}{c}\text { Guided catheter/ } \\
\text { application }\end{array}$ & Availability & Cost & Applicators & $\begin{array}{c}\text { Catheter } \\
\text { reconstruction }\end{array}$ \\
\hline CT & Good & $\begin{array}{c}\text { Useful, limited } \\
\text { by radiation hazard }\end{array}$ & $\begin{array}{c}\text { Commonly } \\
\text { available }\end{array}$ & Moderate & $\begin{array}{c}\text { Cheap, cause } \\
\text { artefact }\end{array}$ & Good \\
\hline MRI & Excellent & $\begin{array}{c}\text { Useful, limited } \\
\text { by availability }\end{array}$ & $\begin{array}{c}\text { Uncommonly } \\
\text { available }\end{array}$ & High & Costly, fragile & Good \\
\hline USG & Good to excellent & $\begin{array}{c}\text { Useful, artifacts can } \\
\text { pose problems }\end{array}$ & $\begin{array}{c}\text { Can be made } \\
\text { commonly } \\
\text { available }\end{array}$ & Low & Cheap & Good
\end{tabular}

CT - computed tomography, MRI - magnetic resonance imaging, USG - ultrasonography

izing radiation, has no nephrotoxicity, and can provide diagnostic information comparable to CT and MRI [31].

With the expanding use and availability of USG, the impression that USG is a subjective assessment may not hold true in the near future. A study focusing on training Indian surgery residents in use of abdominal USG for 2 months showed that it was not difficult to learn with achievement of similar proficiency as that of a radiology resident following the training [32]. Studies from USA have reported on increasing utilization of USG by anesthetists for regional block procedures, and its use has rapidly entered the field of acute pain medicine and regional anesthesia and interventional pain medicine over the last decade, raring to become the standard of practice in due course of time [33]. Ultrasonography training may become progressively easier with reducing cost of machines as well as ease of preparation of low cost phantoms for USG [34]. A summary of the authors' understanding comparing CT, MR and USG has been tabulated in Table 1.

\section{Limitations}

Morton has pointed on several limitations of the use of USG in prostate brachytherapy, and these apply equally to other sites as well. It needs a shielded operating room or the ability to deliver anesthesia in an HDR bunker. As image quality can be degraded by calcification, bleeding, or the shadowing effects of implanted catheters, it is important to get high-quality USG images. Ultrasonography is prone to distortion, sensitive to the temperature, and constitution of the medium, therefore requiring regular calibration. Radiation oncologists are unfamiliar with using USG, hence training for the same should be introduced to increase the confidence level of future doctors. The TRUS probe itself may cause a decreasing space between the vaginal wall or prostate and anterior rectal wall, with a possibility of higher dose in the contact region, and appropriate precautions need to be taken to get around this possibility. In gynecologic brachytherapy, we can try to use real-time guidance to create space between the posterior vaginal wall and anterior rectum. This can improve the potential therapeutic ratio of brachytherapy [13].

Clinical data using 3-dimensional USG in gynecological cancer and its use as a sole modality for volumetric treatment planning in cervical cancer are limited, due to poorer reproducibility and sub-optimal visualization of critical structures [35]. High-dose-rate prostate brachytherapy has circumvented some of these challenges, however further work is clearly needed especially for cervix and other sites. Considering the benchmark that USG has attained in prostate brachytherapy and accepting that most of the limitations are technical, scientific advancements in appropriate directions will hopefully solve the issues.

\section{Conclusions}

Ultimately, the ideal treatment process following USG-based catheter insertion would be to perform real-time USG planning and delivery. Recent advancements in USG imaging and planning software of brachytherapy will help us obtain a real-time guidance, and estimate the dose delivery to the insertion position with zero geometric uncertainty. There are some limitations, which need to be addressed.

\section{Acknowledgement}

Dr Susovan Banerjee was deeply influenced by use of USG in prostate cancer brachytherapy by Dr. Gerard Morton, Sunnybrook Cancer Hospitals, Toronto, during educational visit in January 2016.

\section{Disclosure}

Authors report no conflict of interest.

\section{References}

1. European guidelines for quality assurance in radiotherapy. Booklet No. 8; http://www.estro.org/binaries/content/ assets/estro/school/publications/booklet-8---a-practicalguide-to-quality-control-of-brachytherapy-equipment.pdf.

2. Holm HH, Juul N, Pedersen JF et al. Transperineal 125iodine seed implantation in prostatic cancer guided by transrectal ultrasonography. J Urol 1983; 130: 283-286.

3. Morton GC. Prostate high-dose-rate brachytherapy: Transrectal ultrasound based planning, a technical note. Pract Radiat Oncol 2014; 5: 238-240.

4. Lauche O, Delouya G, Taussky D et al. Single-fraction highdose-rate brachytherapy using real-time transrectal ultrasound based planning in combination with external beam radiotherapy for prostate cancer: dosimetrics and early clinical results. J Contemp Brachytherapy 2016; 8: 104-109. 
5. Pieters B, Wijkstra H, van Herk M et al. Contrast-enhanced ultrasound as support for prostate brachytherapy treatment planning. J Contemp Brachytherapy 2012; 4: 69-74.

6. Brascho DJ, Kim RY, Wilson EE. Use of Ultrasonography in Planning Intracavitary Radiotherapy of Endometrial Carcinoma. Radiology 1978; 129: 163-167.

7. Rao PB, Ghosh S. Routine use of ultrasound guided tandem placement in intracavitary brachytherapy for the treatment of cervical cancer - a South Indian institutional experience. J Contemp Brachytherapy 2015; 7: 352-356.

8. Narayan K, van Dyk S, Bernshaw D et al. Ultrasound guided conformal brachytherapy of cervix cancer: survival, patterns of failure, and late complications. J Gynecol Oncol 2014; 25: 206-213.

9. Van Dyk S, Narayan K, Fisher R et al. Conformal brachytherapy planning for cervical cancer using transabdominal ultrasound. Int J Radiat Oncol Biol Phys 2009; 75: 64-70.

10. Stock RG, Chan $K$, Terk $M$ et al. A new technique for performing Syed-Neblett template interstitial implants for gynecologic malignancies using transrectal-ultrasound guidance. Int J Radiat Oncol Biol Phys 1997; 37: 819-825.

11. Sharma DN, Rath GK, Thulkar S et al. Use of transrectal ultrasound for high dose rate interstitial brachytherapy for patients of carcinoma of uterine cervix. J Gynecol Oncol 2010; 21: 12-17.

12. Mahantshetty U, Khanna N, Swamidas J et al. Trans-abdominal ultrasound (US) and magnetic resonance imaging (MRI) correlation for conformal intracavitary brachytherapy in carcinoma of the uterine cervix. Radiother Oncol 2012; 102: 130-134.

13. Kamrava M. Potential role of ultrasound imaging in interstitial image based cervical cancer brachytherapy. J Contemp Brachytherapy 2014; 6: 223-230.

14. Schmid MP, Nesvacil N, Pötter R et al. Transrectal ultrasound for image-guided adaptive brachytherapy in cervix cancer - an alternative to MRI for target definition? Radiother Oncol 2016; 120: 467-472.

15. Petric P, Kirisits C. Potential role of TRAns Cervical Endosonography (TRACE) in brachytherapy of cervical cancer: proof of concept. J Contemp Brachytherapy 2016; 8: 215-220.

16. Strnad V, Ott OJ, Hildebrandt G et al. 5-year results of accelerated partial breast irradiation using sole interstitial multicatheter brachytherapy versus whole-breast irradiation with boost after breast-conserving surgery for low-risk invasive and in-situ carcinoma of the female breast: a randomized, phase 3, non-inferiority trial. Lancet 2016; 387: 229-238.

17. Koh VY, Buhari SA, Tan PW et al. Comparing a volume based template approach and ultrasound guided freehand approach in multicatheter interstitial accelerated partial breast irradiation. J Contemp Brachytherapy 2014; 6: 173-177.

18. DeBiose DA, Horwitz EM, Martinez AA et al. The use of ultrasonography in the localization of the lumpectomy cavity for interstitial brachytherapy of the breast. Int J Radiat Oncol Biol Phys 1997; 38: 755-759.

19. Trifiletti DM, Romano KD, Showalter SL et al. Accelerated partial breast irradiation with brachytherapy: patient selection and technique considerations. Breast Cancer (Dove Med Press) 2015; 7: 211-221.

20. De Jean P, Beaulieu L, Fenster A. Three-dimensional ultrasound system for guided breast brachytherapy. Med Phys 2009; 36: 5099-5106.

21. Poulin E, Gardi L, Fenster A et al. Towards real-time 3D ultrasound planning and personalized 3D printing for breast HDR brachytherapy treatment. Radiother Oncol 2015; 114: 335-338.

22. Christensen AF, Nielsen MB, Engelholm SA. Three-dimensional endoluminal ultrasound-guided interstitial brachytherapy in patients with anal cancer. Acta Radiologica 2008; 49: 132-137.
23. Niehoff P, Kovács G. HDR brachytherapy for anal cancer. J Gastrointest Oncol 2014; 5: 218-222.

24. Niehoff P, Schumacher N, Siebert FA et al. PO-1040: TRUS guided interstitial HDR Brachytherapy combined with RCT for treatment of anal cancer. Radiother Oncol 2014; 111: S160.

25. Becker HD, Harms W, Debus J et al. Brachytherapy of inoperable peripheral lung cancer guided by electromagnetic navigation and endobronchial ultrasound: Feasibility study and confirmation by long-term results at two centres. Chest Meeting Abstracts 2009; 136: 2.

26. Kishi K, Takifujia K, Shiraia S et al. Brachytherapy Technique for Abdominal Wall Metastases of Colorectal Cancer: Ultrasound-Guided Insertion of Applicator Needle and a Skin Preservation Method. Acta Radiologica 2006; 47: 157-161.

27. Minsky B, Botet J, Gerdes H, et al. Ultrasound directed extrahepatic bile duct intraluminal brachytherapy. Int J Radiat Oncol Biol Phys 1992; 23: 165-167.

28. Maier W, Henne K ,Schipper J. Endoscopic ultrasound-guided brachytherapy of head and neck tumours. A new procedure for controlled application. J Laryngol Otol 1999; 113: 41-48.

29. Wolfgang C, Schlegel, Thomas B. New technologies in Radiation Therapy. Springer Germany 2006: 239-241.

30. Fenster A, Parraga G, Bax J. Three-dimensional ultrasound scanning. Interface Focus 2011; 1: 503-519.

31. Wilson SR, Greenbaum LD, Goldberg BB. Contrast-enhanced ultrasound: what is the evidence and what are the obstacles? AJR Am J Roentgenol 2009; 193: 55-60.

32. Sridhara S, Rana KV, Naware $S$ et al. Ultrasound training in surgical residency: Is it feasible? Med J DY Patil Univ 2014; 7: 284-288.

33. Marhofer P, Chan VW. Ultrasound-guided regional anesthesia: current concepts and future trends. Anesth Analg 2007; 104:1265-1269.

34. Khera PS, Keshava SN. An indigenous model for learning ultrasound-guided interventions. The Indian J Radiol Imaging 2014; 24: 132-134

35. Foster W, Froment MA, Noël P et al. Use of 3D-Ultrasound Imaging in Cervical Cancer Brachytherapy: Preliminary Report. Brachytherapy 2015; 14: S11-S106. 